\title{
Falk Symposium Series
}

150. Galle PR, Gerken G, Schmidt WE, Wiedenmann B, eds. Disease Progression and Disease Prevention in Hepatology and Gastroenterology. Falk Symposium 150. 2006

ISBN 1-4020-5109-3

151. Fraser A, Gibson PR, Hibi T, Qian JM, Schölmerich J, eds. Emerging Issues in Inflammatory Bowel Disease. Falk Symposium 151. 2006

ISBN 978-1-4020-5701-4

152. Fockens P, Schulz H-J, Rösch T, Špicčák J, eds. Endoscopy 2006 - Update and Live Demonstration. Falk Symposium 152. 2008 ISBN 978-1-4020-9147-6

153. Dignass A, Rachmilewitz D, Stange E-F, Weinstock JV, eds. Immunoregulation in Inflammatory Bowel Diseases - Current Understanding and Innovation. Falk Symposium 153. 2007

ISBN 978-1-4020-5888-2

154. Adler G, Fiocchi C, Lazebnik LB, Vorobiev GI, eds. Inflammatory Bowel Disease - Diagnostic and Therapeutic Strategies. Falk Symposium 154. 2007

ISBN 978-1-4020-6115-8

155. Keppler D, Beuers U, Leuschner U, Stiehl A, Trauner M, Paumgartner G, eds. Bile Acids: Biological Actions and Clinical Relevance. Falk Symposium 155. 2007

ISBN 978-1-4020-6251-3

156. Blum HE, Cox DW, Häussinger D, Jansen PLM, Kullak-Ublick GA, eds. Genetics in Liver Diseases. Falk Symposium 156. 2007 ISBN 978-1-4020-6393-0

157. Diehl AM, Hayashi N, Manns MP, Sauerbruch T, eds. Chronic Hepatitis: Metabolic, Cholestatic, Viral and Autoimmune. Falk Symposium 157. 2007

ISBN 978-1-4020-6522-4

158. Gasche G, Herrerías Gutiérrez JM, Gassull M, Monterio E, eds. Intestinal Inflammation and Colorectal Cancer. Falk Symposium 158. 2007

ISBN 978-1-4020-6825-6

159. Tözün N, Mantzaris G, Dağlı, Schölmerich J, eds. IBD 2007 - Achievements in Research and Clinical Practice. Falk Symposium 159. 2008

ISBN 978-1-4020-6986-4

160. Ferkolj I, Gangl A, Galle PR, Vucelic B, eds. Pathogenesis and Clinical Practice in Gastroenterology. Falk Symposium 160. 2008

ISBN 978-1-4020-8766-0

161. Carey MC, Gabryelewicz A, Díte P, Keim V, Mössner J, eds. Future Perspectives in Gastroenterology. Falk Symposium 161. 2008

ISBN 978-1-4020-8832-2

162. Bosch J, Lammert F, Burroughs AK, Lebrec D, Sauerbruch T, eds. Liver Cirrhosis: From Pathophysiology to Disease Management. Falk Symposium 162. 2008

ISBN 978-1-4020-8655-7

163. Adler G, Fan DM, Jia JD, LaRusso NF, Owyang C, eds. Chronic Inflammation of Liver and Gut. Falk Symposium 163. 2008

ISBN 978-1-4020-9352-4 
164. Tulassay Z, Dítě P, Krejs GJ, Schölmerich J, Schultz HJ, eds. Intestinal Disorders. Falk Symposium 164. 2009

ISBN 978-1-4020-9590-0

165. Keppler D, Beuers U, Stiehl A, Trauner M, eds. Bile Acid Biology and Therapeutic Actions. Falk Symposium 165. 2009 ISBN 978-1-4020-9643-3

165A. Lieberman DA, Malfertheiner P, Riemann JF, Spechler SJ, eds. Strategies of Cancer Prevention in Gastroenterology. Falk Workshop. 2009

ISBN 978-90-481-2628-6

166. Ell C, Ponchon T, Riemann JF, Sakai P, Yamamoto H, eds. GI Endoscopy - Standards and Innovations. Falk Symposium 166. 2009 ISBN 978-90-481-2748-1

167. Day CP, Galle PR, Lohse AW, Thorgeirsson SS, eds. Liver under Constant Attack - From Fat to Viruses. Falk Symposium 167. 2009

ISBN 978-90-481-2758-0

168. Rogler G, Gassul M, Levine A, López San Román A, eds. IBD in Different Age Groups. Falk Symposium 168. 2009

ISBN 978-3-8055-9273-4

169. Buhr HJ, Kharchenko NV, Siegmund B, Zacharash MP, Zeitz M, eds. Inflammation in the Intestinal Tract: Pathogenesis and Treatment. Falk Symposium 169. 2009

ISBN 978-3-8055-9282-6

170. Travis SPL, Spiller RC, Colombel JF, Holzer P, eds. IBD and IBS: Novel Mechanisms and Future Practice. Falk Symposium 170. 2009

ISBN 978-3-8055-9420-2

171. Day CP, Diehl AM, Lehnert H, Manns MP, Negro F, eds. Liver and Metabolic Syndrome. Falk Symposium 171. 2009 ISBN 978-3-8055-9446-2

171A. Gershwin ME, Lohse AW, Manns MP, Vergani D, eds. Immunology and Liver Disease. Falk Workshop. 2009

ISBN 978-3-8055-9446-2

172. Rogler G, Sandborn W, eds. The Keys to IBD 2010: Treatment, Diagnosis and Pathophysiology. Falk Symposium 172. 2010 ISBN 978-3-8055-9588-9

173. Dítě P, Krejs GJ, Malfertheiner P, Tulassay Z, eds. From Chronic Inflammation to Cancer. Falk Symposium 173. 2010 ISBN 978-3-8055-9653-4

174. Chen CW, Cheng J, Ginès P, Ouyang Q, Schölmerich J, eds. Gut and Liver. Falk Symposium 174. 2010 ISBN 978-3-8055-9672-5

175. Häussinger D, Beuers U, Stiehl A, Trauner M, eds. Bile Acids as Metabolic Integrators and Therapeutics. Falk Symposium 175. 2010

ISBN 978-3-8055-9804-0

176. Blum HE, Hunt RH, Schölmerich J, eds. Environment and Lifestyle - Effects on Disorders of the Digestive Tract. Falk Symposium 176. 2010

ISBN 978-3-8055-9809-5

WS Singer MV, Dooley S, McClain CJ, Zakhari S, eds. Liver and Pancreatic Diseases: Consequences of Chronic Alcoholic Consumption. Falk Workshop Freiburg. 2010

ISBN 978-3-8055-9732-6

WS Schlitt HJ, Benseler V, Hellerbrand C, Loss M, Weiss TS, Wiest R, eds. Liver and Immunology. Falk Workshop Regensburg. 2011 ISBN 978-3-8055-9845-3

177. Schulz HJ, Rösch T, Siersema PD, eds. Endoscopy Live Berlin 2011-Intestinal Disease Meeting. Falk Symposium 177. 2011

ISBN 978-3-8055-9874-3

178. Kruis W, Spiller RC, Papagigoriadis S, Engel A, Kreis ME, eds. Diverticular Disease: A Fresh Approach to a Neglected Disease. Falk Symposium 178. 2011

ISBN 978-3-8055-9878-8

179. D’Haens G, Dignass A, Vermeire S, eds. Revisiting IBD Management: Dogmas to Be Challenged. Falk Symposium 179. 2012

ISBN 978-3-318-02187-5

WS Tiegs G, Lohse AW, Thimme R, Trautwein C, eds. Inflammation and Cancer. Falk Workshop. 2012

ISBN 978-3-318-02185-1

WS Miehlke S, Munch A, eds. Microscopic Colitis - Creating Awareness for an Underestimated Disease. Falk Workshop Basel. 2012 
181. Tilg H, Blumberg RS, Endres S, Kaser A, Manns MP, eds. Innate Immunity in Gastrointestinal Disorder: Basic and Therapeutic Concepts. Falk Symposium 181. 2012

ISBN 978-3-318-02210-0

182. Friess H, Schmid RM, Fried M, van de Velde CJH, eds. Carcinogenesis, Prevention and Treatment of Colorectal Cancer - State of the Art 2012. Falk Symposium 182. 2012

ISBN 978-3-318-02213-1

183. Rogler C, Beglinger C, Colombel J-F, Michetti P, eds. Dealing with Our 'In-Vironment': New Aspects in IBD Pathogenesis and Therapy. Falk Symposium 183. 2012

ISBN 978-3-318-02258-2

184. Häussinger D, Beuers U, Trauner M, eds. XXII International Bile Acid Meeting - Hepatic and Extrahepatic Targets of Bile Acid Signaling. Falk Symposium 184. 2012

185. Ell C, Galle PR, Mössner J, Meyenberger C, Spechler SJ, Yamamoto H, eds. Interfaces and Controversies in Gastroenterology. Falk Symposium 185. 2013

ISBN 978-3-318-02384-8

186. Galle PR, Clavien P-A, Gores GJ, Protzer U, Schuchmann M, eds. Challenges of Liver Cirrhosis and Tumors: Prevent It, Treat It, Manage Consequences. Falk Symposium 186. 2013 ISBN 978-3-318-02384-8

187. Panés J, Ghosh S, Gomollón F, Louis E, eds. Overcoming Challenges in IBD Management. Falk Symposium 187. 2013 ISBN 978-3-318-02468-5

188. Stange EF, Dignass A, Fellermann K, Herrlinger K, eds. Inflammatory Bowel Diseases: Microbiota versus the Barrier. Falk Symposium 188. 2013

ISBN 978-3-318-02538-5

189. Straumann A, Schoepfer A, Simon H-U, eds. Eosinophilic Esophagitis: A Novel Chronic Inflammatory Disease of the GI Tract. Falk Symposium 189. 2013

WS Krejs GJ, Eherer AJ, Pointner R, eds. GERD: Gastroesophageal Reflux Disease. Falk Workshop Graz. 2013 ISBN 978-3-318-02594-1

190. Levine A, Forbes A, Probert C, eds. Challenges in the Care of IBD in Patients of All Ages. Falk Symposium 190. 2013 ISBN 978-3-318-02662-7

191. Chapman RW, Adams DH, Beuers U, Day CP, Manns MP, eds. Liver Diseases in 2013: Advances in Pathogenesis and Treatment. Falk Symposium 191. 2013

ISBN 978-3-318-02665-8

192. Dignass A, Allez M, Danese S, Marteau P, eds. IBD 2014: Thinking Out of the Box. Falk Symposium 192. 2014 ISBN 978-3-318-02806-5

193. Mulder CJJ, Schuppan D, eds. Celiac Disease and Other Small Bowel Disorders. Falk Symposium 193. 2014 ISBN 978-3-318-03014-3

194. Häussinger D, Beuers U, Keitel V, Trauner M, eds. XXIII International Bile Acid Meeting: Bile Acids as a Signal Integrators and Metabolic Modulators. Falk Symposium 194. 2014

ISBN 978-3-318-05436-1

WS Gerbes AL, Jaeschke HW, eds. The Challenge of Drug-Induced Liver Injury (DILI). Falk Workshop Freiburg. 2015

195. Gerbes AL, Bosch J, Pinzani M, Wong F, eds. Challenges and Management of Liver Cirrhosis. Falk Symposium 195. 2015 ISBN 978-3-318-05448-4 